Military Technical College Kobry El-Kobbah, Cairo, Egypt

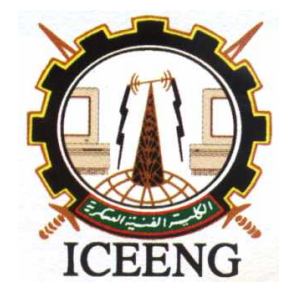

\author{
$6^{\text {th }}$ International Conference \\ on Electrical Engineering \\ ICEENG 2008
}

\title{
Modified LDA classifier in multi resolution wavelet domain for multi-pose face recognition
}

By

I Gede Pasek Suta Wijaya,***

Keiichi Uchimura*

Zhencheng $\mathrm{Hu}^{*}$

\section{Abstract:}

This paper presents multipose faces recognition. The proposed scheme is based on holistic information of face image and small modification of classical LDA (modified LDA) classifier. The holistic information called as facial features is obtained by multiresolution wavelet analysis. The modified LDA (MLDA) classifier that works based on multivariate analysis classifies the facial features to a person's class. The objectives of the proposed method are to create a compact and meaningful facial features without removing significant face image information, to build a simple classification technique which can well classify face images to a person's class, to make the M-LDA-based training system to solve the retraining problem of the PCA and LDA based recognition system, to reduce the high memory space requirement of classical LDA and PCA, and to compare the effectiveness of proposed method to established LDA based recognition systems such as RLDA, DLDA, and SLDA. The result shows that the proposed method gives good enough performance i.e. high enough success rate, short time processing, and small enough EER compare to establish LDA. In addition, the wavelet transforms is an efficient way for reducing the dimensional size of original image.

\section{Keywords:}

facial features, matching, wavelet, LDA, eigenface

* Computer Science and Electrical Engineering Graduate School of Science and Technology, Kumamoto University Kumamoto-Shi, Japan

** Electrical Engineering Dept., Engineering Faculty, Mataram University Indonesia 


\section{Introduction:}

Human face image recognition is an active research area in image processing applications because there are many potential applications, which cover human computer interactions, forensics, surveillance, and security systems. Recently, the demand of biometrics system as security system has been increasing significantly to substitute password and PIN security system. The main weakness of the password and PIN system is little bit easy cracked them by guessing because $81 \%$ of users use the same password, PIN, and 30\% write them down or store them in a file. Therefore, face is one of the biometrics systems that can be implemented as security system for substituting the password and PIN system.

This paper proposes an alternative face recognition system, which is based on holistic or global information of face image and MLDA. The holistic information of face image called as facial features is obtained by multiresolution wavelet analysis of entire image without geometrical normalization and localization. The function of the MLDA is to classify the facial features to a person's class. The main aims of this method are to solve large computational costs, high memory space requirements and retraining problems LDA based face recognition.

\section{Previous Work:}

The previous works related to our approach are face recognition based on holistic or global approach as described in Refs. [1,2,3,4,5,9]. Ref. [4] describes face recognition based on wavelet packet tree analysis for frontal view of human faces under roughly constant illumination. The facial features were built by implementing wavelet- packet tree analysis of bounding box face and then calculating the mean and variance of sixteen matrixes wavelet coefficients. That approach does not work for non-frontal and small variation faces view and needs constant illumination to make the face-bounding box. Ref. [5] describes face recognition based on combination of DCT analysis and face localization technique for finding the global information of face image, but it requires eyes coordinate, which have to input manually, to perform geometrical normalization. The global face information was created by keeping small part of big magnitude values of DCT coefficients.

The mostly related approach to our system is face recognition based on the LDA and its variations as described in Ref. [2,3,6]. Ref. [2] proposed a combination of D-LDA and F-LDA to cover the weakness of classical LDA. It only solves the poor discriminatory and singularity problem. Ref. [3] implemented DCT to reduce data dimensional and only small part of DCT coefficients is analyzed by LDA. Ref. [6] implemented the 
wavelet transforms to reduce the dimension of face image, employ a regulation scheme for the within-scatter matrix, and use optimization procedure. It was reported that the Daubechies (Db-6) was implemented to filter image to resolution 29 x 23. However, those methods have limitations: large computational cost, high memory spaces requirement, and retraining problems.

In our method, we implement multiresolution wavelets analysis for reducing the original data dimensional and MLDA classifier for classifying the face class without geometrical normalization and bounding box processing. It is difficult to compare our results with previous works because their time consuming were rarely reported and the tests were carried out with different databases. Therefore, our approach results will be compared to establish LDA, which has been tested with data from four face databases.

\section{LDA based face recognition:}

\subsection{Classical LDA}

The main purpose of LDA analysis is to find a linear transformation such that feature clusters are most separable after the transformation. It can be achieved by the betweenclass scatter matrix $S_{b}$ and the within-class scatter matrix $S_{w}$ analysis, as explained in Ref. [3]. The class separation is measured by the ratio of determinant of the $S_{b}$ matrix to the $S_{\mathrm{w}}$ matrix using the equation below.

$E=\arg \max _{E} \frac{\left|E^{T} S_{b} E\right|}{\left|E^{T} S_{w} E\right|}$

Where $E=\left[e_{1}, e_{2}, e_{3}, \ldots, e_{m}\right]$ is set of eigen-vectors corresponding to $m$ largest eigenvalues $\lambda_{i}$ which satisfy the equation: $S_{b} e_{i}=\lambda_{i} S_{W} e_{i}, i=1,2,3, \ldots, m$. The eigenvectors and eigen-values are obtained by computing the inverse of $S_{b}$ and then solving the eigen problem of $\mathrm{S}_{\mathrm{W}}^{-1} \mathrm{~S}_{\mathrm{b}}$ matrix. Finally, the projection of the linear discriminant function is given by:

$$
\mathrm{Y}_{\mathrm{i}}(\mathrm{C})=\mathrm{E}^{\mathrm{T}}\left(\mathrm{C}_{\mathrm{i}}-\overline{\mathrm{m}}_{\mathrm{i}}\right)
$$

The intrinsic problem of above algorithm is the singularity problem of scatter matrix due to the high data dimensional and small number of training samples called as small size problem (SSS). Some methods have been proposed to solve that problem such as DLDA, RLDA, sand PCA+LDA as described in Refs. [2,7,8]. However, those methods still require large computational costs and the retraining problems. 


\subsection{M-LDA for Face Recognition}

In order to solve retraining problem, we propose the M-LDA approach. The M-LDA is based on assumption that the matrix scatter has small dimension and the covariance of the training images is multivariate normal distribution. The proposed algorithm is described as below.

Let define a big matrix, $Q=\left[\mathrm{X}_{\mathrm{i}}\right]_{\mathrm{i}=1}^{\mathrm{n}}$, containing $\mathrm{n}$ classes with each class $\mathrm{X}_{\mathrm{i}}=\left[\mathrm{X}_{\mathrm{i}, \mathrm{j}}\right]_{\mathrm{j}=1}^{\mathrm{m}}$ consisting $m$ column vector of facial features, where $m$ is number of member class $X_{i}$. Next, the mean of each class is easily determined and then placed it into mean matrix as $\mathrm{M}=\left[\mu_{1}, \mu_{2}, \mu_{3}, \ldots, \mu_{\mathrm{c}}\right]$, where $\mu_{\mathrm{i}}=\mathrm{E}\left(\mathrm{X}_{\mathrm{i}}\right)=\left(1 / \mathrm{m}_{\mathrm{i}}\right) \sum_{\mathrm{j}=1}^{\mathrm{m}_{\mathrm{i}} \mathrm{x}_{\mathrm{i}, \mathrm{j}}}$. Finally, we can determine the global covariance using the with-in class $\left(\mathrm{S}_{\mathrm{w}}\right)$ equation.

$\mathrm{S}_{\mathrm{w}}=\mathrm{C}_{\mathrm{g}}=\sum_{\mathrm{i}=1}^{\mathrm{c}} \sum_{\mathrm{x}_{\mathrm{j}} \in \mathrm{C}_{\mathrm{i}}}\left(\mathrm{x}_{\mathrm{j}}-\mu_{\mathrm{i}}\right)\left(\mathrm{x}_{\mathrm{j}}-\mu_{\mathrm{i}}\right)^{\mathrm{T}}$

If $\mathrm{C}_{\mathrm{g}}$ is multivariate normal distribution, we can classify of each facial features to person's classes using the equation below.

$F_{c}=\max \left[g_{1}(x), g_{2}(x), g_{3}(x), \ldots, g_{m}(x)\right]$

Where $\mathrm{g}_{\mathrm{i}}(\mathrm{x})$ is given by:

$g_{i}(x)=\mu_{i} C^{-1} x^{T}-0.5 \mu_{i} C^{-1} \mu_{i}^{T}$

The equation (5) is derived from maximum a posteriori (MAP) discriminant, as describe below:

$g_{i}(x)=P\left(\varpi_{i} \mid x\right)=\frac{P\left(x \mid \varpi_{i}\right) P\left(\varpi_{i}\right)}{P(x)}$

$\mathrm{g}_{\mathrm{i}}(\mathrm{x})=\frac{1}{\left(2 \pi^{\mathrm{n} / 2}\right)\left|\mathrm{C}_{\mathrm{i}}\right|^{1 / 2}} \exp \left\{-1 / 2\left(\mathrm{x}-\mu_{\mathrm{i}}\right)^{\mathrm{T}} \mathrm{C}_{\mathrm{i}}^{-1}\left(\mathrm{x}-\mu_{\mathrm{i}}\right)\right\} \mathrm{P}\left(\varpi_{\mathrm{i}}\right) \frac{1}{\mathrm{P}(\mathrm{x})}$

where $\mathrm{P}(\mathrm{x})$ is total probability of $\mathrm{x}$. By eliminating the constant term and taking the natural $\log$, it becomes.

$\mathrm{g}_{\mathrm{i}}(\mathrm{x})=-\frac{1}{2}\left(\mathrm{x}-\mu_{\mathrm{i}}\right)^{\mathrm{T}} \mathrm{C}_{\mathrm{i}}^{-1}\left(\mathrm{x}-\mu_{\mathrm{i}}\right)-\frac{1}{2} \log \left(\left|\mathrm{C}_{\mathrm{i}}\right|\right)+\log \left(\mathrm{P}\left(\varpi_{\mathrm{i}}\right)\right)$

As mentioned previously that all classes have identical covariance and the same prior probability, the equation (8) can be simplified as below: 
$g_{i}(x)=-\frac{1}{2} x C^{-1} x^{T}+\mu_{i} C^{-1} x^{T}-\frac{1}{2} \mu_{i} C^{-1} \mu_{i}{ }^{T}$

By keeping just the terms dependent on $\mu_{\mathrm{i}}$ and $\mathrm{C}$, the equation (5) is obtained.

This algorithm has some advantages for classifying the face image class:

1. It is simple because it does not require the eigen-values and eigen-vectors analysis.

2. It can solve the retraining problem as illustrated: firstly, when a new class added to the system, the M-LDA calculates the mean and the covariance of its class; secondly, the newest mean is placed into the matrix $M$; and finally, the previous covariance is updated by adding it with the newest class covariance.

3. The computation complexity is less than the PCA and LDA computation complexity because of not requiring eigen analysis.

The weakness of M-LDA is singularity problem due to the high data dimensional and small number of training samples. To overcome the singularity problem, we implement frequency analysis to reduce the data dimensional as explained in the next section.

\section{Facial features extraction:}

In this research, we develop a holistic approach for facial features extraction based on multiresolution DWT analysis in the entire image without geometrical normalization and bounding process. The multiresolution wavelet analysis is performed by implementing repeatedly classical DWT called as filter bank decomposition, as shown in Fig. 1. The A, H, V, and D are calculated by equation (10).

$$
\begin{aligned}
& \mathrm{A}=\left[\mathrm{h} *[\mathrm{~h} * \mathrm{f}]_{\mathrm{x}} \downarrow_{2}\right]_{\mathrm{y}} \downarrow_{2} \\
& \mathrm{H}=\left[\mathrm{g} *[\mathrm{~h} * \mathrm{f}]_{\mathrm{x}} \downarrow_{2}\right]_{\mathrm{y}} \downarrow_{2} \\
& \mathrm{~V}=\left[\mathrm{h} *[\mathrm{~g} * \mathrm{f}]_{\mathrm{x}} \downarrow_{2}\right]_{\mathrm{y}} \downarrow_{2} \\
& \mathrm{D}=\left[\mathrm{g} *[\mathrm{~g} * \mathrm{f}]_{\mathrm{x}} \downarrow_{2}\right]_{\mathrm{y}} \downarrow_{2}
\end{aligned}
$$
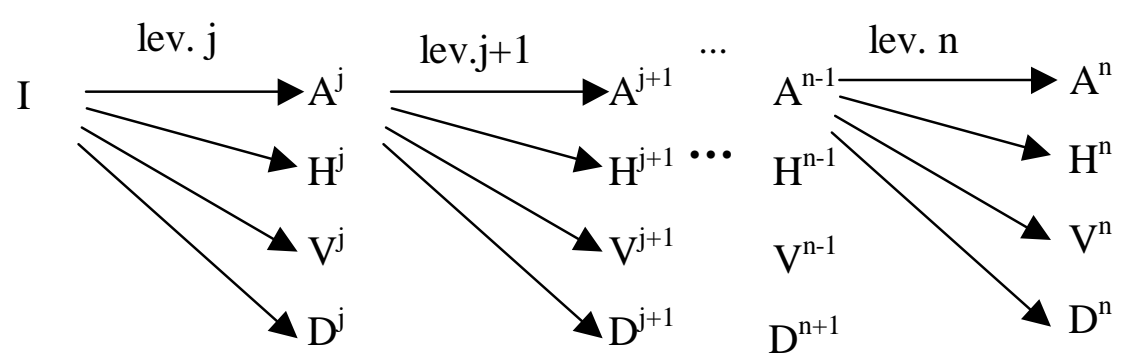

Figure (1): filter-bank wavelet decomposition. 
where, $*$ denote convolution, $\downarrow_{2}$ represent down sampling for $\mathrm{x}$ and $\mathrm{y}$ direction, $\mathrm{g}$ and $\mathrm{h}$ are high and low pass filter respectively. In order to make simple and fast decomposition process, we apply two different Daubachies wavelets basis, namely Db4 and Db1. First, Db4 basis decomposes face images until level 2 and it just return the approximation coefficients. Second, the Db1 basis decomposes the Db4's approximation coefficient until maximum level. This decomposition returns the wavelet coefficients as shown in Fig. 2. From these coefficients, the compact and meaningful facial features called as holistic information are created by three steps: firstly, convert the frequency domain coefficients to vector using row ordering technique; secondly, sort the vector descending using quick sort algorithm, finally truncate a small number of vector elements (i.e., less then 100 elements). Those processes are performed on both training and query (probe) face images. However, in the training process, those are performed one time.

Consider the compact facial features, if they are reconstructed into the face images, the reconstructed face images will be different. However, we can still understand that they are the face images, as shown in the Fig. 3(a). Meanwhile, if the compact facial features are removed the reconstructed face images are exactly different and we do not know that they are face images at all, as shown in the Fig. 3(b). This illustration proves that the most information of image exists in low frequency components.

The compact facial features can be used as a basis of multipose face recognition because they consist of dominant of frequency components of the face image. It means that the facial features of any face pose variations in a single face are identical. It can be proved by calculating the correlation coefficients of both compact facial features and original image of any face pose variations in a single face, as shown in Fig. 4. It shows that the correlations of compact facial features are almost the same for all face poses. However, the correlations of the original have large different values for all face poses.

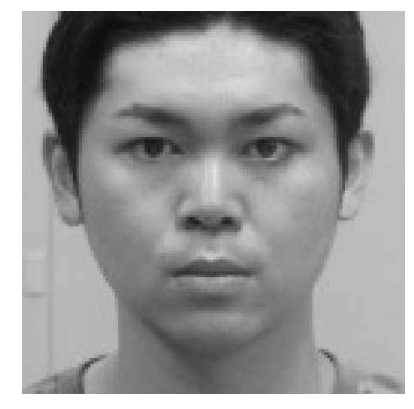

(a)

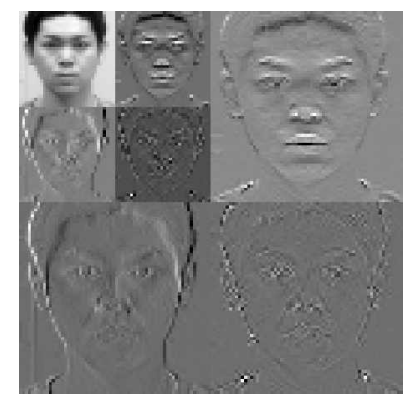

(b)

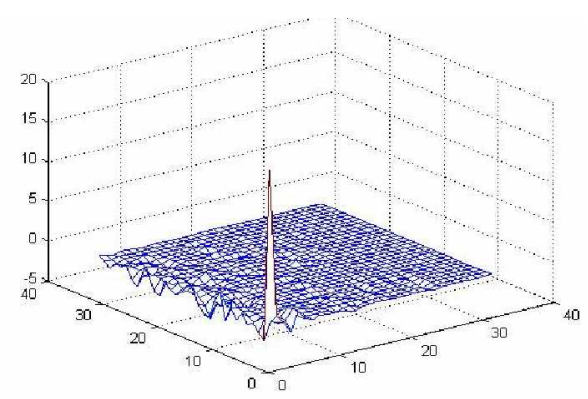

(c)

Figure (2): the output of multiresolution wavelet analysis: (a) original image, (b) the first step decomposition coefficients, (c) the second step decomposition coefficients. 

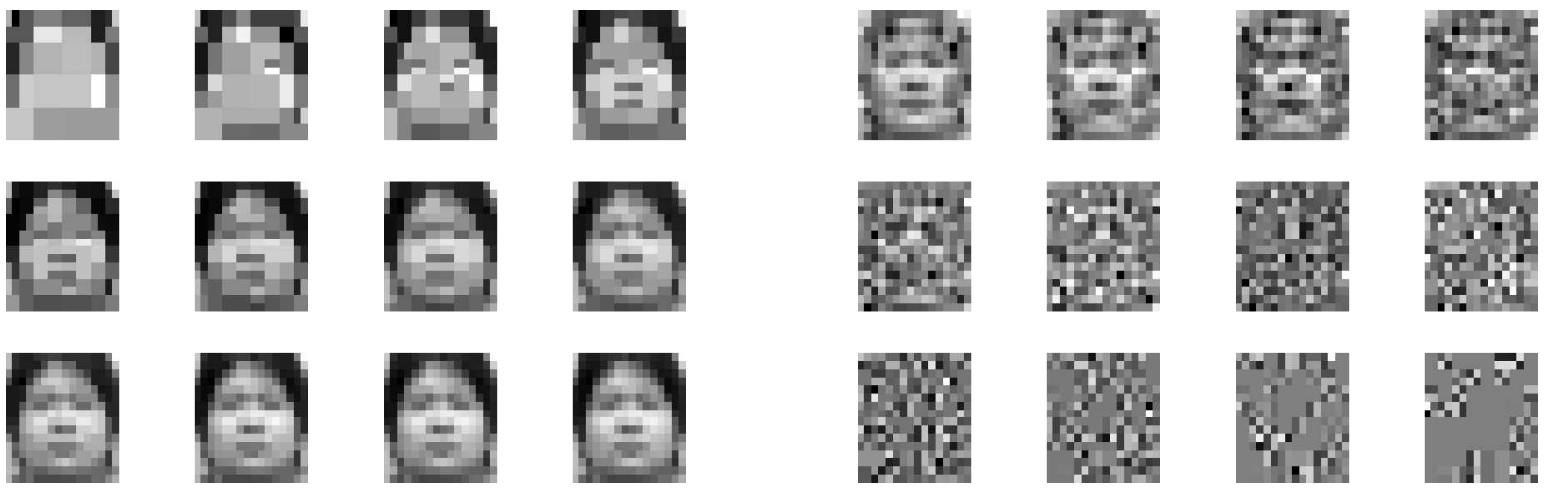

(a)

(b)

Figure (3): (a) the reconstructed images of the compact facial features that the size ranges from 16 elements (top-left) until 225 elements (bottom-right), (b) the reconstructed images when the compact facial features are removed.

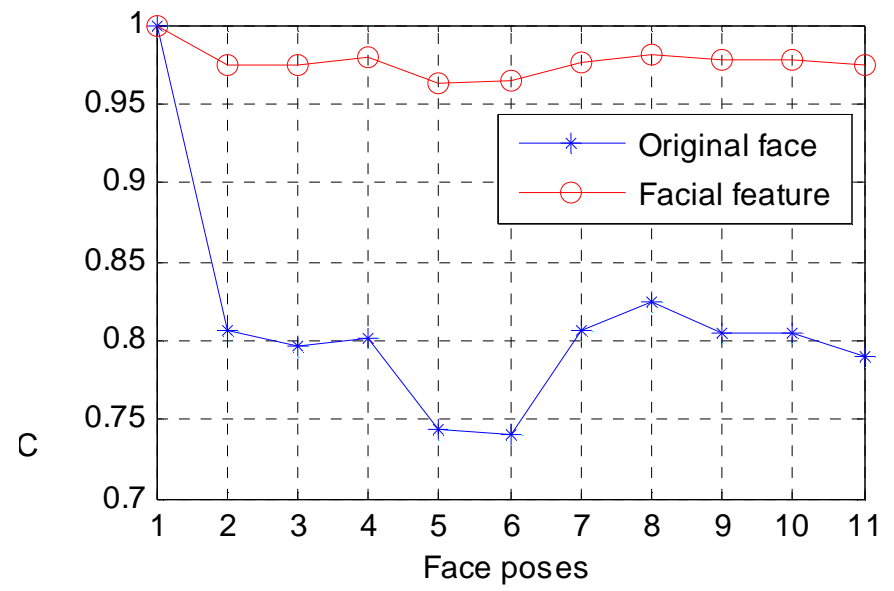

Figure (4): the correlation coefficients comparison between the original image and the compact facial of eleven face poses of India face database.

\section{Face recognition algorithm:}

The proposed face recognition algorithm can be illustrated briefly as Fig. (5). There are three main process in this algorithm, namely preprocessing process, training process, and recognition process. The preprocessing unit consists of color space transformation, equalization, and multiresolution wavelet analysis. In this research, NTSC (YIQ) color space is implemented for to convert the color image (RGB) to gray component (Y). Actually, there are two main function of preprocessing process: firstly, to decrease the effect of the non-uniform lighting condition on image face, which is performed using standard equalization, secondly, to create compact facial features, using multiresolution wavelet transforms as described in section 4 . In the training process, the compact facial features set is analyzed by MLDA for finding mean of each class and the global covariance and then save them in database as a meaningful data for face classification. 


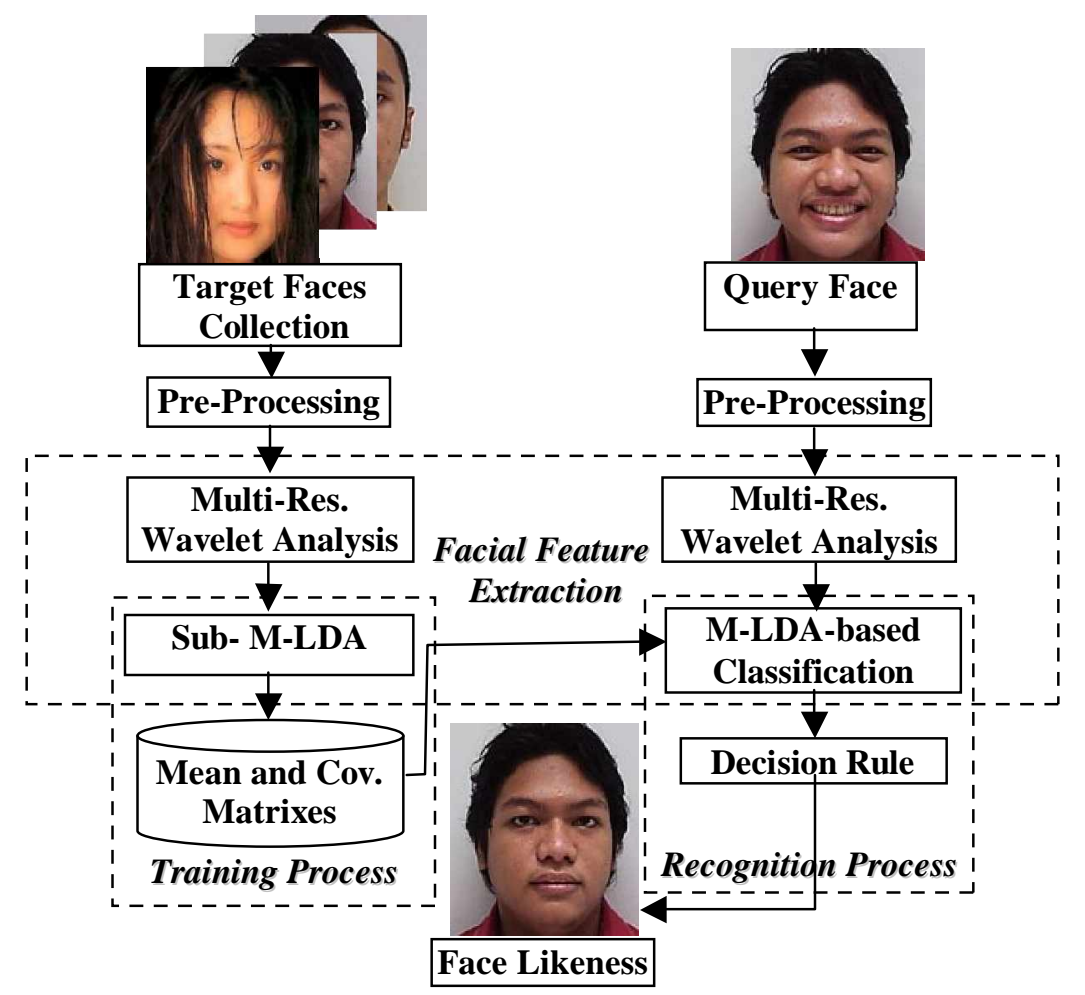

Figure (5): brief face recognition algorithm.

Finally, we calculate the similarity between the input facial features and the training facial features set using MLDA based classifier. In this case, the minimum score is concluded as the best likeness.

\section{Exsperiment and Result:}

The experiments were carried out using data from four face databases: ITS-Lab. Kumamoto University database, EE-UNRAM database, India database [10], and ORL database [11]. Each database has special characteristics. The ITS-Lab database consists of 48 people and each person has 10 pose orientations as shown in Fig. 6. The face images were taken by Konica Minolta camera series VIVID 900 under varying lighting condition. The India database consists 61 people ( 22 women and 39 men), each person has eleven pose orientations: looking front, looking left, looking right, looking up, looking up towards left, looking up towards right, and looking down. Indian database also included the emotions: neutral, smile, laughter, sad/disgust. The EE-UNRAM database consists of 40 people and each person has 8 pose orientations: looking front, looking left about $30^{\circ}$, looking right about $30^{\circ}$, looking up, looking down, and wearing accessory such as glasses. The ORL database was taken at different times, under varying the lighting conditions, facial with different expressions (open/closed eyes, smiling/not smiling) and facial details (glasses/no glasses). All of the images were 


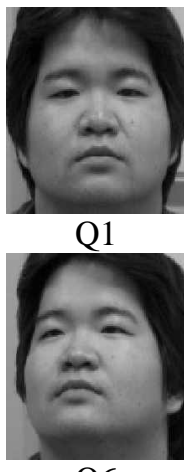

Q6

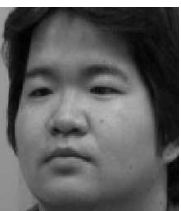

Q2

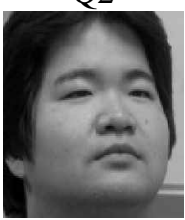

Q7

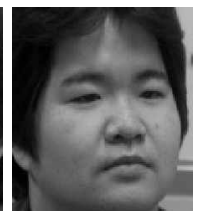

Q3

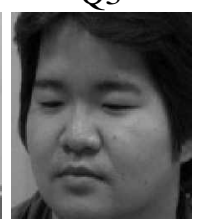

Q8

(a)

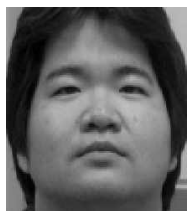

Q4

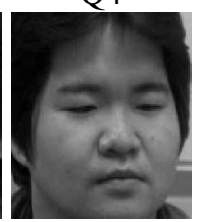

Q9

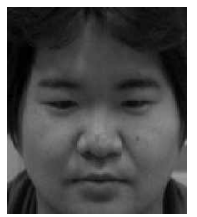

Q5

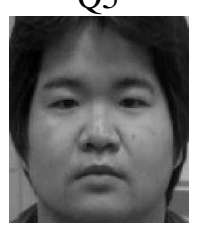

Q10

Figure (6): Example of face poses of ITS-Lab. face database.

taken against a dark homogeneous background. The faces of the subjects are in an upright, frontal position (with tolerance for some side movement). The ORL database is a grayscale face database that consists of 40 people, mainly male.

In order to know the performance of the proposed method, some experiments were done using data from previously mentioned databases. All of experiments were performed in image size of $128 \times 128$ pixel, the facial vector size of 49 elements, and 5 training face per class except the UNRAM database was 4 training face per class. They were chosen based on the information in Ref. [9]. The first experiment investigated the accuracy of the proposed method in tested databases and compared with the establish LDA such as DLDA, RLDA, and SLDA. The experimental result can be shown in Fig. (7).

The result shows that the proposed method gives little bit better accuracy for all databases because the facial features contain not only the low frequency components but also small number of high frequency components, as explained in section 4. Regarding
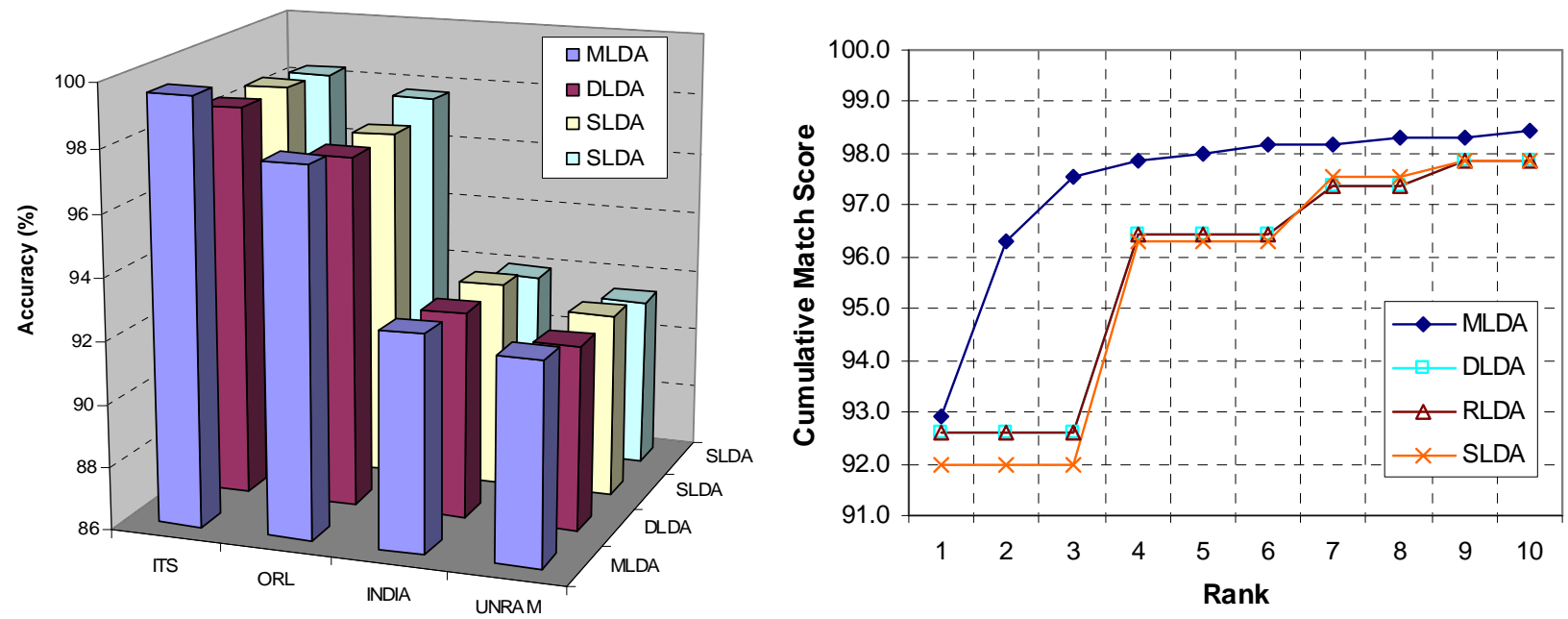

Figure (7): the recognition accuracy (a) and CMS (b) comparison of LDA based face recognition. 
to the Cumulative Match Score, the proposed method show better performance than established LDA in the same facial features size. In this case, the MLDA was show better accuracy and CMS because the LDA discriminate the facial features like the Mahalanobis distance classifier. However, the MLDA has problems in terms of singularity. In this research, it is solved by reducing the original data size using multiresolution wavelet transforms (MWT). By MWT the original data can be compacted to 49 elements of 16384 elements, it means the original data is compressed by about $99.66 \%$.

The second experiment was performed to investigate both training time and querying time and compare with the established LDA's time consumption. The results shows that the proposed method requires shorter training and querying times than LDA's time process, as show on Fig 9(a). Regarding to the retraining problem the MLDA method require very short time when new class is added to the system as shows in Fig. 9(b). This result can be achieved because the MLDA does not require eigen value analysis and the covariance analysis do not depend on the global mean of the training set.

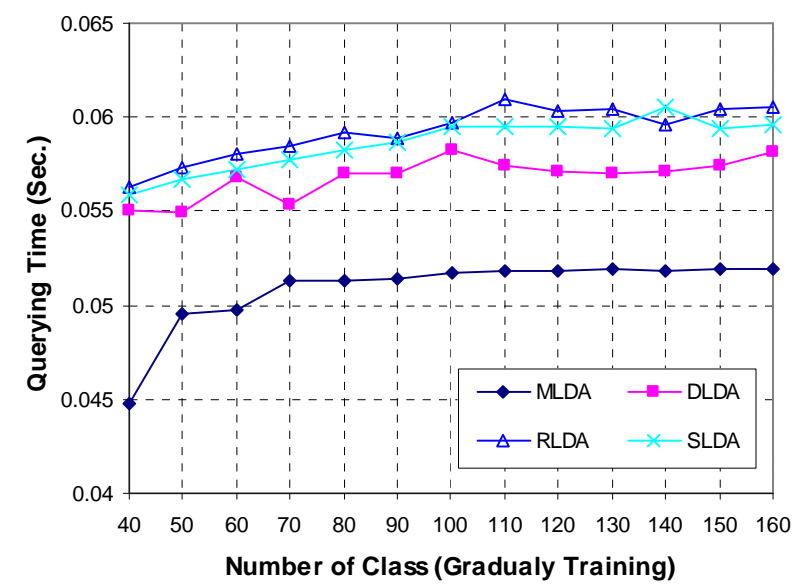

(a)

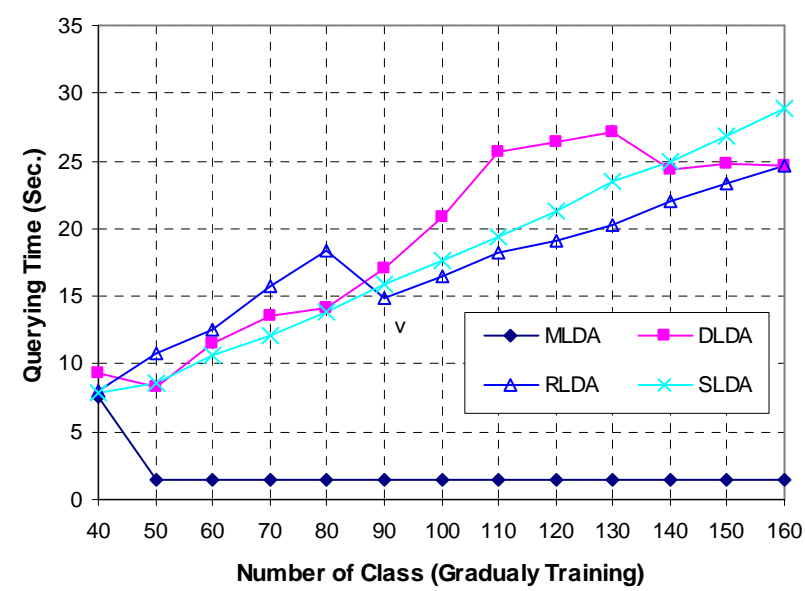

(b)

Figure (9): the time consumption comparison of LDA based method when the training was performs gradually: (a) querying time, (b) training time.

There are two aspects, which can be used to justify a good recognition system: first, how well the system can match image from the same people; second, how well the system distinguish images from different people [9]. Therefore, the last test was performs in order to know the performance of the proposed method in term of ROC analysis. The main parameters that want to know are equal error rate (ERR), false acceptance rate (FAR), and false rejection rate (FAR). The detail explanations of the ROC can be found in Ref. [9]. The system which performs perfect classification is denoted by $100 \%$ true positive rate and $0 \%$ false positive rate or the value of ERR is small or close to zero. The last experiment was performed on all databases, 49 elements facial features size, and 5 faces training per class. For the tested databases, the ROC 


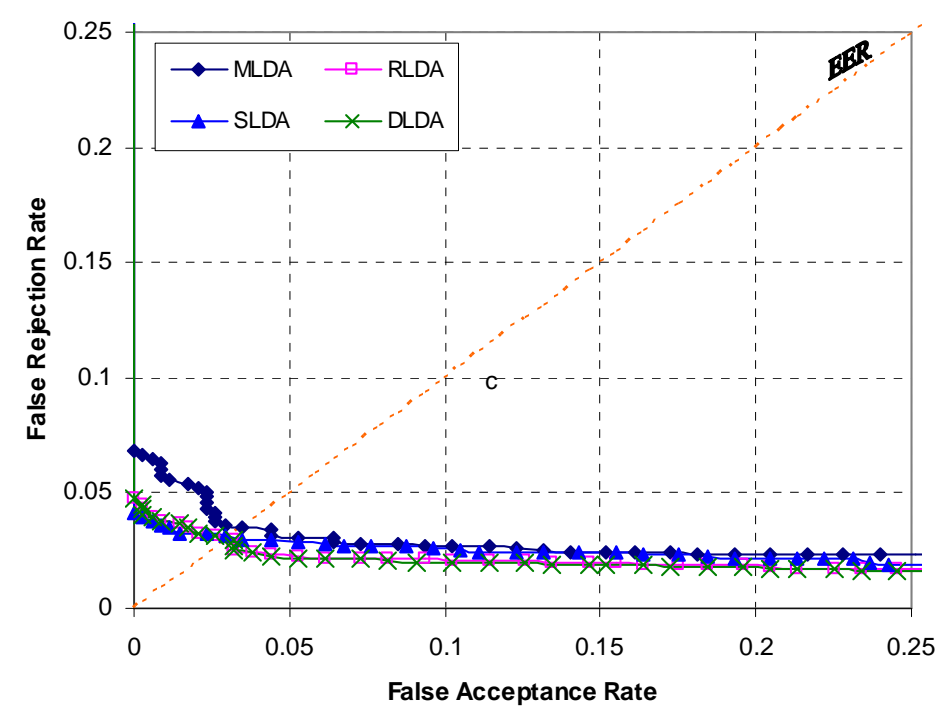

Figure (10): ROC analysis of LDA based face recognition.

curve show that the EER of the proposed method is almost the same with EER of the established LDA, however our method gives better success rate, requires less time consumption, and can solved the retraining problem.

All of the experimental results show that the proposed method has good performance, robust to tested face databases, and need short training and querying times. This performance can be achieved because facial features have good low-frequency resolution representation. In this case, the low frequency component is good enough for face image representation because most information of signal can be found in low frequency component, as shown in Fig. (3). It can be described that if an image is transformed to frequency domain and the high frequency components are removed, the reconstruction image will loss a little significant information. This phenomenon was successfully used for signal compression. Moreover, wavelet decomposition property gives advantage for features extraction, such as it has good capability to separate information signal to low frequency components and their coefficients are uncorrelated with other frequency indices.

The multiresolution wavelet decomposition is an efficient way of reducing the original data dimensional. In this paper, we show that the original data size can be reduced about $99.66 \%$ of original size (i.e., 49 elements of 16384 elements), while the success rate is high enough. Also note that facial features vector is built by fast wavelet transforms and the M-LDA based classification just need mean of each face class and global covariance to classify face image to a person's class. Computational complexity of wavelet decomposition is linear with the number $(N)$ of computed coefficients $(\mathrm{O}(\mathrm{N}))$, where $\mathrm{N}$ number of data. Therefore, our method needs short training and querying times 


\section{Conclusions:}

The proposed method is alternative face recognition because it gives good enough performance i.e. high enough success rate, short time processing, and small enough EER. In addition, the multiresolution wavelet transforms is an efficient way for reducing the dimensional size of original image and it requires a short decomposition time. Moreover, the proposed method can cover the retraining problem as explained in section 3.3 and is proved in the second experiment (Fig. 9(b)). However, this method has to test in large databases size for knowing its accuracy, CMS, and EER. Moreover, this process needs some improvements, such as considering other color space component and implementing the hybrid facial features (i.e DCT and DWT) to make the powerful facial features. Next, the research will focus on face image preprocessing analysis and finding optimum classification method in order to increase the accuracy.

\section{References:}

[1] Chellappa, R., Wilson, C., and Shirohey, S., Human and Machine recognition of faces: A survey, In Proc. IEEE, vol. 83, no. 5, P. 705-740, 1995.

[2] Lu, J., Plataniotis K.N., and Venetsanopoulus A.N., "Face Recognition Using LDABased Algorithmn", IEEE Transactions on Neural Networks, vol. 14, no. 1, P. 195200, 2003.

[3] Chen, W., Er, Meng J., and Wu S., PCA and LDA in DCT Domain, ELSEVIER Pattern Recognition Letter, 26, P. 2474-2482, 2005

[4] Garcia C., Zikos G., and Tziritas G., Wavelet Packet Analysis for Face Recognition, Image and Vision Computing, 18, P. 289-297, 2000

[5] Hafed, Ziad M., and Levine, Martin D., Face Recognition Using the Discrete Cosine Trasnforms, International Journal of Computer Vision, 43(3), P. 167-188, 2001

[6] Dai, Dao-Qing and Yuen P.C., Wavelet Based Discriminant Analysis for Face Recognition, ELSEVIER Applied Mathematics and Computation, 175, P. 307-318, 2006

[7] H. Yu, J. Yang, A direct LDA algorithm for high-dimensional data-with application to face recognition, Pattern Recognition 34, P. 2067-2070., 2001

[8] Gao H, Davis James W., Why direct LDA is not equivalent to LDA, ELSEVIER Pattern Recognition Letter, 39, P. 1002-1006, 2005

[9] Wijaya, I G.P., Uchimura, K., and Hu, Z., Face Recognition Based on Holistic Information and Minimun Mahalanobis Classifier, Proceedings of the ACCV 2007 Workshop Subspace 2007 Tokyo, P. 53-60, November 2007

[10] http://vis-ww.cs.umass.edu/ vidit/IndianFaceDatabase

[11] http://www.cl.cam.ac.uk/research/dtg/attarchive/facedatabase.html 